\title{
GRÖBNER-COHERENT RINGS AND MODULES
}

\author{
ROHIT NAGPAL AND ANDREW SNOWDEN
}

\begin{abstract}
Let $R$ be a graded ring. We introduce a class of graded $R$-modules called Gröbner-coherent modules. Roughly, these are graded $R$-modules that are coherent as ungraded modules because they admit an adequate theory of Gröbner bases. The class of Gröbner-coherent modules is formally similar to the class of coherent modules: for instance, it is an abelian category closed under extension. However, Gröbner-coherent modules come with tools for effective computation that are not present for coherent modules.
\end{abstract}

\section{Contents}

1. Introduction 1

2. Background on coherence 2

3. Gröbner bases 2

4. Gröbner coherence 4

5. Further results 6

6. Relation between different notions of coherence $\quad 7$

$\begin{array}{ll}\text { References } & 7\end{array}$

\section{INTRODUCTION}

Let $R$ be a graded ring. The purpose of this paper is to isolate a particular class of graded $R$-modules: the Gröbner-coherent modules. Roughly speaking, these are graded $R$-modules that are coherent as ungraded $R$-modules but not simply by happenstance: they have a "reason" for their coherence related to the grading (that reason basically being an adequate theory of Gröbner bases). Formally, the class of Gröbner-coherent modules over $R$ behaves similarly to the class of coherent modules: for instance, it is an abelian category and closed under extensions. However, Gröbner-coherent modules enjoy an advantage over coherent modules in that they come with effective computation procedures. For instance, if $R$ is a Gröbner-coherent ring (meaning Gröbner-coherent as a module over itself) and one can compute with finitely presented graded $R$-modules then one can also compute with finitely presented (ungraded) $R$-modules.

Our original motivation for developing the theory of Gröbner-coherence was in relation to our study of divided power algebras [NS]. Let $\mathbf{D}$ be the divided power algebra in one variable over a noetherian ring $\mathbf{k}$. Typically, $\mathbf{D}$ is not noetherian. We show that $\mathbf{D}$ is a Gröbnercoherent ring. As a consequence, if one can compute with finitely generated $\mathbf{k}$-modules then one can also compute with finitely presented D-modules.

Date: June 13, 2016.

AS was supported by NSF grants DMS-1303082 and DMS-1453893. 


\section{BACKGROUND ON COHERENCE}

Let $R$ be a ring. An $R$-module $M$ is coherent if it is finitely generated and every finitely generated submodule is finitely presented; clearly, a coherent module is finitely presented. The category of coherent modules is abelian and closed under extensions. The ring $R$ is coherent if it is coherent as a module over itself. In this case, every finitely presented module is coherent. Whenever we refer to coherence of a graded ring or module we ignore the grading.

Suppose now that $R$ is a graded ring. Then a graded $R$-module $M$ is graded-coherent if it is finitely generated and every finitely generated homogeneous submodule is finitely presented. Once again, a graded-coherent module is finitely presented, and the category of graded-coherent modules is abelian and closed under extensions. The ring $R$ is gradedcoherent if it is graded-coherent as a module, and then every finitely presented graded module is graded-coherent.

\section{GRÖBNER BASES}

Gröbner bases are typically employed to study ideals in a polynomial ring, or, more generally, submodules of free modules over polynomial rings. However, the ideas apply equally well to study inhomogeneous submodules of graded modules over an arbitrary graded ring. In this section, we develop the theory of Gröbner bases in this greater generality.

Let $R$ be a graded ring, and let $M$ be a graded $R$-module. We assume $R$ is supported in non-negative degrees and that $M_{n}=0$ for $n \ll 0$. Let $x=\sum_{n \in \mathbf{Z}} x_{n}$ be a nonzero element of $M$ with $x_{n} \in M_{n}$. We define the degree of $x$, denoted $\operatorname{deg}(x)$, to be the maximal $n$ such that $x_{n} \neq 0$. We define the initial term of $x$, denoted in $(x)$, to be $x_{n}$ where $n=\operatorname{deg}(x)$. Given an (inhomogeneous) submodule $N$ of $M$, we define the initial submodule, denoted $\operatorname{in}(N)$, to be the homogeneous submodule generated by $\operatorname{in}(x)$ over all nonzero $x \in N$.

Definition 3.1. Let $N$ be a submodule of a graded module $M$. A collection $\left\{x_{i}\right\}_{i \in I}$ of elements in $N$ is a Gröbner basis for $N$ if the $\operatorname{in}\left(x_{i}\right)$ generate $\operatorname{in}(N)$ as an $R$-module.

Definition 3.2. Let $N$ be a submodule of a graded module $M$, let $\left\{x_{i}\right\}_{i \in I}$ be a collection of elements of $N$, and let $y$ be another element of $N$. An expression $y=\sum_{i \in I} a_{i} x_{i}$ with $a_{i} \in R$ is reduced if $\operatorname{deg}\left(a_{i}\right)+\operatorname{deg}\left(x_{i}\right) \leq \operatorname{deg}(y)$ for all $i$.

Proposition 3.3. Let $M$ be a graded $R$-module, let $N$ be a submodule, and let $\left\{x_{i}\right\}_{i \in I}$ be a collection of elements of $N$. Let $\sum_{i \in I} c_{i, j}$ in $\left(x_{i}\right)=0$, for $j$ in an index set $J$, be a homogeneous generating set for the module of syzygies of the $\operatorname{in}\left(x_{i}\right)$, and put $z_{j}=\sum_{i \in I} c_{i, j} x_{i}$. Then the following are equivalent:

(a) The $x_{i}$ form a Gröbner basis for $N$.

(b) Every element of $N$ has a reduced expression in terms of the $x_{i}$.

(c) The $x_{i}$ generate $N$ and each $z_{j}$ has a reduced expression in terms of the $x_{i}$.

Proof. (a) $\Longrightarrow$ (b). Let $\mathrm{S}_{n}$ be the statement "every element of $N$ of degree $\leq n$ has a reduced expression in terms of the $x_{i}$." For $n \ll 0$, the statement $\mathrm{S}_{n}$ is obviously true. Now suppose that $\mathrm{S}_{n-1}$ is true, and let us prove $\mathrm{S}_{n}$. Thus let $y \in N$ be an element of degree $n$. We then have an expression $\operatorname{in}(y)=\sum_{i \in I} a_{i} \operatorname{in}\left(x_{i}\right)$ where $\operatorname{deg}\left(a_{i}\right)+\operatorname{deg}\left(x_{i}\right)=\operatorname{deg}(y)$. Let $y^{\prime}=y-\sum_{i \in I} a_{i} \operatorname{in}\left(x_{i}\right)$. Then $y^{\prime} \in N$ and $\operatorname{deg}\left(y^{\prime}\right) \leq n-1$, so by $\mathrm{S}_{n-1}$ we have an expression $y^{\prime}=\sum b_{i} x_{i}$ with $\operatorname{deg}\left(b_{i}\right)+\operatorname{deg}\left(x_{i}\right) \leq n-1$. Thus $y=\sum\left(a_{i}+b_{i}\right) x_{i}$ is reduced expression for $y$, and $\mathrm{S}_{n}$ follows. We conclude that $\mathrm{S}_{n}$ holds for all $n$ by induction, and so (b) holds. 
(b) $\Longrightarrow($ a). Let $y$ be a nonzero element of $N$, and let us show that in $(y)$ can be generated by the in $\left(x_{i}\right)$. Using (b), we have an expression $y=\sum_{i \in I} a_{i} x_{i}$ where $\operatorname{deg}\left(a_{i}\right)+\operatorname{deg}\left(x_{i}\right) \leq$ $\operatorname{deg}(y)$. Let $J \subset I$ be the set of indices $i$ such that $a_{i} x_{i}$ has degree equal to $\operatorname{deg}(y)$. Then $\operatorname{in}(y)=\sum_{i \in J} \operatorname{in}\left(a_{i} x_{i}\right)=\sum_{i \in J} \operatorname{in}\left(a_{i}\right)$ in $\left(x_{i}\right)$. Thus the $\left\{x_{i}\right\}$ form a Gröbner basis, and so (a) holds.

(b) $\Longrightarrow$ (c) is immediate, (b) implies that the $x_{i}$ generate $N$, and explicitly states that the $z_{j}$ admit reduced expressions.

$(\mathrm{c}) \Longrightarrow(\mathrm{b})$. Let $y \in N$ be given. Let $y=\sum_{i \in I} a_{i} x_{i}$ be an expression for $y$ in terms of the $x_{i}$ 's with $\delta=\max _{i \in I}\left(\operatorname{deg}\left(a_{i}\right)+\operatorname{deg}\left(x_{i}\right)\right)$ minimal. We claim $\delta=\operatorname{deg}(y)$, and so the expression is reduced. Assume not. Write $a_{i}=a_{i}^{\prime}+a_{i}^{\prime \prime}$ where $a_{i}^{\prime \prime}$ is the degree $\delta-\operatorname{deg}\left(x_{i}\right)$ piece of $a_{i}$ (and $a_{i}^{\prime}$ has smaller degree). Then $\sum_{i \in I} a_{i}^{\prime \prime} \operatorname{in}\left(x_{i}\right)=0$. This is a homogeneous syzygy of the in $\left(x_{i}\right)$, and so we have an expression in terms of the c's: there exist homogeneous elements $b_{j} \in R$ satisfying $\operatorname{deg}\left(b_{j}\right)+\operatorname{deg}\left(c_{i, j}\right)=\operatorname{deg}\left(a_{i}^{\prime \prime}\right)$ such that $a_{i}^{\prime \prime}=\sum_{j \in J} b_{j} c_{i, j}$. We have

$$
y=\sum_{i \in I} a_{i} x_{i}=\sum_{i \in I} a_{i}^{\prime} x_{i}+\sum_{i \in I} \sum_{j \in J} b_{j} c_{i, j} x_{i}=\sum_{i \in I} a_{i}^{\prime} x_{i}+\sum_{j \in J} b_{j} z_{j} .
$$

Using the reduced expression for the $z$ 's, this gives an expression $y=\sum_{i \in I} d_{i} x_{i}$ with $\max \left(\operatorname{deg}\left(d_{i}\right)+\operatorname{deg}\left(x_{i}\right)\right)<\delta$, a contradiction. Thus $\delta=\operatorname{deg}(y)$ as claimed, and (b) holds.

The above proposition leads to Buchberger's algorithm for finding a Gröbner basis. Let $X=\left\{x_{i}\right\}_{i \in I}$ be a generating set for $N \subset M$. The algorithm proceeds as follows:

(a) Compute the set $Z=\left\{z_{j}\right\}_{j \in J}$ as in the proposition.

(b) If each $z_{j}$ has a reduced expression, output $X$ and terminate.

(c) Otherwise, replace $X$ with $X \cup Z$ and return to step (a).

If the algorithm terminates, then its output is a Gröbner basis. If the input set $X$ is finite and $M$ is graded-coherent, then $X$ will remain finite after each step, and so if the algorithm terminates it will produce a finite Gröbner basis. Note that in the usual description of Buchberger's algorithm, one does not add the $z_{j}$ 's to $X$, but their remainders after applying the generalized division algorithm. This is more efficient, but makes no theoretical difference.

In general, analysis of Buchberger's algorithm can be quite difficult. However, there is one situation that we can analyze easily:

Proposition 3.4. Let $M$ be a graded module, $N$ a submodule, and $\left\{x_{i}\right\}_{i \in I}$ a generating set for $N$. Suppose that there exists an integer $\delta \geq 0$ such that every element $y \in N$ admits an expression of the form $y=\sum_{i \in I} a_{i} x_{i}$ with $\operatorname{deg}\left(a_{i}\right)+\operatorname{deg}\left(x_{i}\right) \leq \operatorname{deg}(y)+\delta$. Then Buchberger's algorithm terminates after at most $\delta$ steps.

Proof. This is like (c) $\Longrightarrow$ (b) from the previous proposition: each step in the algorithm lets us reduce $\delta$ by at least one, so we eventually get down to $\delta=0$.

The following proposition shows how Gröbner bases can be used to compute syzygies.

Proposition 3.5. Let $M$ be a graded module, let $N$ be a submodule, and let $\left\{x_{i}\right\}_{i \in I}$ be a Gröbner basis for $N$. Let $\sum_{i \in I} c_{i, j} \operatorname{in}\left(x_{i}\right)=0$, for $j \in J$, be a homogeneous generating set for the module of syzygies of the $\operatorname{in}\left(x_{i}\right)$, let $z_{j}=\sum_{i \in I} c_{i, j} x_{i}$, and let $z_{j}=\sum_{i \in I} d_{i, j} x_{i}$ be a reduced expression. Let $\Phi: \bigoplus_{i \in I} R e_{i} \rightarrow N$ be the map defined by $\Phi\left(e_{i}\right)=x_{i}$. We regard $e_{i}$ as homogeneous of degree $\operatorname{deg}\left(x_{i}\right)$. Let $r_{j}=\sum_{i \in I}\left(c_{i, j}-d_{i, j}\right) e_{i}$. Then $\left\{r_{j}\right\}_{j \in J}$ is a Gröbner basis for $K=\operatorname{ker}(\Phi)$. 
Proof. By definition, $\operatorname{deg}\left(c_{i, j}\right)+\operatorname{deg}\left(x_{i}\right)=d_{j}$ is independent of $i$. We have $\operatorname{deg}\left(z_{j}\right)<d_{j}$, and so $\operatorname{deg}\left(d_{i, j}\right)+\operatorname{deg}\left(x_{i}\right)<d_{j}$ as well. Thus in $\left(r_{j}\right)=\sum_{i \in I} c_{i, j} e_{i}$. Now, suppose that $s=\sum_{i \in I} a_{i} e_{i}$ is an element of $K$. Thus $\sum_{i \in I} a_{i} x_{i}=0$. Let $\delta=\operatorname{deg}(s)=\max _{i \in I}\left(\operatorname{deg}\left(a_{i}\right)+\operatorname{deg}\left(x_{i}\right)\right)$, and write $a_{i}=a_{i}^{\prime}+a_{i}^{\prime \prime}$ where $a_{i}^{\prime \prime}$ is the homogeneous degree $\delta-\operatorname{deg}\left(x_{i}\right)$ piece of $a_{i}$. Then $\sum_{i \in I} a_{i}^{\prime \prime} \operatorname{in}\left(x_{i}\right)=0$ is a homogeneous syzygy, and so there are homogeneous element $b_{j} \in R$ with $\operatorname{deg}\left(b_{j}\right)+\operatorname{deg}\left(c_{i, j}\right)=\operatorname{deg}\left(a_{i}^{\prime \prime}\right)$ such that $a_{i}^{\prime \prime}=\sum_{j \in J} c_{i, j} b_{j}$. We have

$$
s-\sum_{j \in J} b_{j} r_{j}=\sum_{i \in I} a_{i}^{\prime} e_{i}+\sum_{i \in I} \sum_{j \in J} b_{j} d_{i, j} e_{i} .
$$

Note that $\operatorname{deg}\left(a_{i}^{\prime}\right)+\operatorname{deg}\left(e_{i}\right)=\operatorname{deg}\left(a_{i}^{\prime}\right)+\operatorname{deg}\left(x_{i}\right)<\delta$ and $\operatorname{deg}\left(b_{j}\right)+\operatorname{deg}\left(d_{i, j}\right)+\operatorname{deg}\left(e_{i}\right)<$ $\operatorname{deg}\left(b_{j}\right)+d_{j}=\operatorname{deg}\left(b_{j}\right)+\operatorname{deg}\left(c_{i, j}\right)+\operatorname{deg}\left(x_{i}\right) \leq \delta$. Thus the equation above shows that $\operatorname{deg}\left(s-\sum_{j \in J} b_{j} r_{j}\right)<\delta$ and so $\operatorname{in}(s)$ is generated by the $\operatorname{in}\left(r_{j}\right)$. This implies that $\left\{r_{j}\right\}_{j \in J}$ is a Gröbner basis for $K$.

\section{GRÖBNER COHERENCE}

The primary definitions are:

Definition 4.1. Let $R$ be a graded ring. We say that a graded $R$-module $M$ is Gröbnercoherent if it is graded-coherent and every finitely generated inhomogeneous submodule admits a finite Gröbner basis.

Definition 4.2. We say that a graded $\operatorname{ring} R$ is Gröbner-coherent if it is so as a module over itself.

Remark 4.3. The two conditions in Definition 4.1 (namely, graded-coherent and every submodule admits a finite Gröbner basis) play off of each other nicely, as computations with Gröbner bases often reduce to computations with leading terms, and the graded-coherence ensures that such computations behave well.

Proposition 4.4. Let $M$ be a Gröbner-coherent graded module. Then $M$ is coherent.

Proof. Let $N$ be a finitely generated inhomogeneous submodule of $M$. Let $\left\{x_{i}\right\}_{i \in I}$ be a finite Gröbner basis for $N$, and let $\Phi: \bigoplus_{i \in I} R e_{i} \rightarrow N$ be the surjection defined by $\Phi\left(e_{i}\right)=x_{i}$. It follows from Proposition 3.5, that $\operatorname{ker}(\Phi)$ has a finite Gröbner basis (note that the gradedcoherence of $M$ implies that the set $J$ in Proposition 3.5 is finite), and so $\operatorname{ker}(\Phi)$ is finitely generated. This shows that $N$ is finitely presented, completing the proof.

Proposition 4.5. Let $M$ be a Gröbner-coherent $R$-module and let $M^{\prime} \subset M$ be a finitely generated homogeneous submodule. Then $M^{\prime}$ and $M / M^{\prime}$ are both Gröbner-coherent.

Proof. It follows from basic properties of graded-coherence that $M^{\prime}$ and $M / M^{\prime}$ are gradedcoherent. If $N$ is a finitely generated submodule of $M^{\prime}$, then it is also one of $M$, and thus admits a finite Gröbner basis. Thus $M^{\prime}$ is Gröbner-coherent.

Now let $N$ be a finitely generated submodule of $M / M^{\prime}$, and let $\widetilde{N}$ be its inverse image in $M$, which is finitely generated. Let $\left\{\widetilde{x}_{i}\right\}_{i \in I}$ be a finite Gröbner basis for $\widetilde{N}$, and let $x_{i}$ be the image of $\widetilde{x}_{i}$ in $N$. Let $y \in N$ and let $\widetilde{y}$ be a lift of $y$ to $\widetilde{N}$ with $\operatorname{deg}(y)=\operatorname{deg}(\widetilde{y})$. Let $\widetilde{y}=\sum_{i \in I} a_{i} \widetilde{x}_{i}$ be a reduced expression. Then $y=\sum_{i \in I} a_{i} x_{i}$ is also a reduced expression, and so $\left\{x_{i}\right\}_{i \in I}$ is a finite Gröbner basis for $N$. This shows that $M / M^{\prime}$ is Gröbner-coherent.

Proposition 4.6. Let $M^{\prime} \subset M$ be graded $R$-modules such that $M^{\prime}$ and $M / M^{\prime}$ are Gröbnercoherent. Then $M$ is Gröbner-coherent. 
Proof. It follows from basic properties of graded-coherence that $M$ is graded-coherent. Let $N \subset M$ be a finitely generated submodule, and let $\bar{N}$ be its image in $M / M^{\prime}$. Let $\left\{\bar{x}_{i}\right\}_{i \in I}$ be a finite Gröbner basis for $\bar{N}$, and let $x_{i} \in N$ be a lift of $\bar{x}_{i}$. Note that we cannot necessarily pick $x_{i}$ to have the same degree as $\bar{x}_{i}$. Let $\delta=\max _{i \in I}\left(\operatorname{deg}\left(x_{i}\right)-\operatorname{deg}\left(\bar{x}_{i}\right)\right)$. Since $\bar{N}$ is coherent, the kernel $K$ of the map $N \rightarrow \bar{N}$ is a finitely generated submodule of $M^{\prime}$. Let $\left\{x_{j}^{\prime}\right\}_{j \in J}$ be a Gröbner basis for $K$.

Now let $y$ be an element of $N$, and let $\bar{y}$ be its image in $\bar{N}$. Let $\bar{y}=\sum_{i \in I} a_{i} \bar{x}_{i}$ be a reduced expression, so that $\operatorname{deg}\left(a_{i}\right)+\operatorname{deg}\left(\bar{x}_{i}\right) \leq \operatorname{deg}(\bar{y}) \leq \operatorname{deg}(y)$. We have $\operatorname{deg}\left(a_{i}\right)+\operatorname{deg}\left(x_{i}\right) \leq$ $\operatorname{deg}(y)+\delta$. Put $y^{\prime}=y-\sum_{i \in I} a_{i} x_{i}$. This is an element of $K$ satisfying $\operatorname{deg}\left(y^{\prime}\right) \leq \operatorname{deg}(y)+\delta$. Let $y^{\prime}=\sum_{j \in J} a_{i}^{\prime} x_{j}^{\prime}$ be a reduced expression, so that $\operatorname{deg}\left(a_{i}^{\prime}\right)+\operatorname{deg}\left(x_{j}^{\prime}\right) \leq \operatorname{deg}(y)+\delta$. We thus have an expression $y=\sum_{i \in I} a_{i} x_{i}+\sum_{j \in J} a_{j}^{\prime} x_{j}^{\prime}$ where $\operatorname{deg}\left(a_{i}\right)+\operatorname{deg}\left(x_{i}\right) \leq \operatorname{deg}(y)+\delta$ and $\operatorname{deg}\left(a_{j}^{\prime}\right)+\operatorname{deg}\left(x_{j}^{\prime}\right) \leq \operatorname{deg}(y)+\delta$. This expression shows that the $x_{i}$ and $x_{j}^{\prime}$ generate $N$, and it follows from Proposition 3.4 that Buchberger's algorithm applied to this generating set stops after at most $\delta$ steps, producing a finite Gröbner basis for $N$. Thus $M$ is Gröbnercoherent.

Corollary 4.7. A finite direct sum of Gröbner-coherent modules is Gröbner-coherent.

Corollary 4.8. The category of Gröbner-coherent $R$-modules is an abelian subcategory of the category of all graded $R$-modules, and is closed under extension.

Proof. Suppose $f: M \rightarrow N$ is a map of Gröbner-coherent modules. Since the category of graded-coherent modules is abelian, we know that kernel, cokernel, and image of $f$ are graded-coherent. In particular, these objects are finitely generated. Hence $\operatorname{ker}(f), \operatorname{coker}(f)$ and $\operatorname{im}(f)$ are Gröbner-coherent by Proposition 4.5. The statement about extensions follows from Proposition 4.6.

Proposition 4.9. Let $R$ be Gröbner-coherent and let $M$ be a graded $R$-module. Then $M$ is Gröbner-coherent if and only if $M$ is finitely presented.

Proof. Suppose that $M$ is finitely presented, and write $M=F / K$ where $F$ is a finite rank free module and $K$ is a finitely generated submodule. Then $F$, being a sum of shifts of $R$, is Gröbner-coherent by Corollary 4.7, and so $M$ is Gröbner-coherent by Proposition 4.5. Conversely, a Gröbner-coherent module (over any ring) is coherent, and thus finitely presented.

The following proposition shows that one can effectively compute a Gröbner basis for an inhomogeneous submodule of a Gröbner-coherent module using Buchberger's algorithm, starting from any set of generators.

Proposition 4.10. Let $M$ be a Gröbner-coherence $R$-module, let $N$ be a finitely generated inhomogeneous submodule of $M$, and let $x_{1}, \ldots, x_{n}$ be a set of generators for $N$. Then Buchburger's algorithm applied to $x_{1}, \ldots, x_{n}$ terminates after finitely many steps and yields a Gröbner basis for $N$.

Proof. Since $M$ is Gröbner-coherent, $N$ admits a finite Gröbner basis, say $y_{1}, \ldots, y_{m}$. Since the $x_{i}$ generate $N$, we can write $y_{i}=\sum_{j=1}^{n} a_{i, j} x_{j}$ for scalars $a_{i, j} \in R$. Let $\delta$ be the maximum value of $\operatorname{deg}\left(a_{i, j}\right)+\operatorname{deg}\left(x_{j}\right)-\operatorname{deg}\left(y_{i}\right)$ over all $i$ and $j$. Now, let $z \in N$ be an arbitrary element. Since $\left\{y_{i}\right\}$ forms a Gröbner basis, we have a reduced expression $z=\sum_{i=1}^{n} b_{i} y_{i}$. This gives $z=\sum_{j=1}^{m} c_{j} x_{j}$ with $c_{j}=\sum_{i=1}^{n} a_{i, j} b_{i}$. We have

$$
\operatorname{deg}\left(c_{j}\right)+\operatorname{deg}\left(x_{j}\right) \leq \operatorname{deg}\left(a_{i, j}\right)+\operatorname{deg}\left(b_{i}\right)+\operatorname{deg}\left(x_{j}\right) \leq \operatorname{deg}(z)+\delta .
$$


Thus Buchberger's algorithm applied to $x_{1}, \ldots, x_{n}$ terminates after at most $\delta$ steps by Proposition 3.4 .

\section{Further RESUlts}

The following result gives a potentially useful way of establishing Gröbner-coherence: once graded-coherence is known, Gröbner-coherence is, in a sense, local.

Proposition 5.1. Let $R$ be a graded ring and let $\mathbf{k}=R_{0}$. Let $M$ be a graded-coherent $R$-module. Then $M$ is Gröbner-coherent if and only if $M_{\mathfrak{m}}$ is Gröbner-coherent for each maximal ideal $\mathfrak{m}$ of $\mathbf{k}$.

Proof. First, suppose $M$ is Gröbner-coherent and let $\mathfrak{m}$ be a maximal ideal of $\mathbf{k}$. Let $N$ be a finitely generated submodule of $M_{\mathfrak{m}}$. Then there is a finitely generated module $N^{\prime}$ of $M$ such that $N=N_{\mathfrak{m}}^{\prime}$. Let $\left\{x_{i}\right\}_{i \in I}$ be a finite Gröbner basis of $N^{\prime}$ and let $y_{i}$ be the image of $x_{i}$ under the natural map $f: N^{\prime} \rightarrow N_{\mathfrak{m}}^{\prime}=N$. Let $y \in \operatorname{in}(N)$. Then there is an element $x \in N^{\prime}$ of the same degree as $y$ such that $y=f(x)$. Let $x=\sum_{i \in I} a_{i} x_{i}$ be a reduced expression. Then $y=\sum_{i \in I} f\left(a_{i}\right) y_{i}$ is a reduced expression as well. This shows that $\left\{y_{i}\right\}_{i \in I}$ is a Gröbner basis of $N$.

Conversely, suppose $M_{\mathfrak{m}}$ is Gröbner-coherent for each maximal ideal $\mathfrak{m}$ of $\mathbf{k}$. Let $N$ be a finitely generated inhomogeneous submodule. Suppose that $\left\{y_{i}\right\}_{i \in I}$ is a finite Gröbner basis for $N_{\mathfrak{m}}$ for some maximal ideal $\mathfrak{m}$. Multiplying by an element $s \in \mathfrak{m}$ if necessary, we may assume that each $y_{i}$ are of the form $f\left(x_{i}\right)$ for some $x_{i} \in N$ where $f$ is the localization map $N \rightarrow N_{\mathfrak{m}}$, and by adding in finitely many elements we may assume that $\left\{x_{i}\right\}_{i \in I}$ generate $N$. Let $\left\{z_{j}\right\}_{j \in J}$ be as in Proposition 3.3. Since $M$ is graded-coherent $J$ can be assumed to be finite. Each $z_{j}$ admits a reduced expression in terms of the $x_{i}$ after localizing at $\mathfrak{m}$. Since there are only finitely many scalars involved, it follows that $z_{j}$ admits such an expression after inverting a single element $s \notin \mathfrak{m}$. Thus by Proposition 3.3, the image of $\left\{x_{i}\right\}_{i \in I}$ under the localization $f_{s}: N \rightarrow N[1 / s]$ define a Gröbner basis for $N[1 / s]$.

Since $M_{\mathfrak{m}}$ is Gröbner-coherent for each $\mathfrak{m}$, the above argument shows that there are $s_{1}, \ldots, s_{n}$ generating the unit ideal and sets $\left\{x_{i}^{k}\right\}_{i \in I_{k}} \subset N$ for $1 \leq k \leq n$ such that for each $k$ the set $\left\{f_{s_{k}}\left(x_{i}^{k}\right)\right\}_{i \in I_{k}}$ is a finite Gröbner basis of $N\left[1 / s_{k}\right]$ and $\left\{x_{i}^{k}\right\}_{i \in I_{k}}$ generates $N$. We claim that $\bigcup_{1 \leq k \leq n}\left\{x_{i}^{k}\right\}_{i \in I_{k}}$ is a Gröbner basis of $N$. To see this let $y \in N$. Multiplying a reduced expression of $f_{s_{k}}(y)$ in terms of $f_{s_{k}}\left(x_{i}^{k}\right)$ by a large enough power $s_{k}^{n_{k}}$ we can obtain a reduced expression $\sum_{i \in I^{k}} a_{i}^{k} x_{i}^{k}$ for $s_{k}^{n_{k}} y$. Since $s_{1}, \ldots, s_{n}$ generate the unit ideal there are $c_{k}$ satisfying $\sum_{k=1}^{n} c_{k} s_{k}^{n_{k}}=1$. Now $\sum_{k=1}^{n} \sum_{i \in I^{k}} c_{k} a_{i}^{k} x_{i}^{k}$ is a reduced expression for $y$. This shows that $\cup_{1 \leq k \leq n}\left\{x_{i}^{k}\right\}_{i \in I_{k}}$ is a Gröbner basis of $N$, completing the proof.

The next results show that Gröbner properties behave well along flat maps.

Proposition 5.2. Let $R \rightarrow S$ be a flat map of graded rings, let $M$ be a graded $R$-module, let $N \subset M$ be an inhomogeneous submodule, and let $\left\{x_{i}\right\}_{i \in I}$ be a Gröbner basis for $M$. Then $\left\{1 \otimes x_{i}\right\}_{i \in I}$ is a Gröbner basis for $S \otimes_{R} N \subset S \otimes_{R} M$.

Proof. Let $\sum_{i \in I} c_{i, j} \operatorname{in}\left(x_{i}\right)=0$, for $j$ in an index set $J$, be a homogeneous generating set for the module of syzygies of the in $\left(x_{i}\right)$. This syzygy module is simply the kernel $K$ of the map $R^{\oplus I} \rightarrow M$ sending the $i$ th basis vector to $\operatorname{in}\left(x_{i}\right)$. Since $R \rightarrow S$ is flat, it follows that the map $S^{\oplus I} \rightarrow S \otimes_{R} M$ has kernel $S \otimes_{R} K$. Thus the relations $\sum_{i \in I} c_{i, j}\left(1 \otimes \operatorname{in}\left(x_{i}\right)\right)=0$, for $j \in J$, form a homogeneous generating set for the module of syzygies of the $1 \otimes \operatorname{in}\left(x_{i}\right)$. Let $z_{j}$ be as in Proposition 3.3. Since the $x_{i}$ form a Gröbner basis, each $z_{j}$ admits a reduced expression 
in terms of the $x_{i}$. It follows that $1 \otimes z_{j}$ also admits a reduced expression in terms of the $1 \otimes x_{i}$, and thus the $1 \otimes x_{i}$ form a Gröbner basis by Proposition 3.3.

Proposition 5.3. Let $\left\{R_{i}\right\}_{i \in I}$ be a directed system of Gröbner-coherent graded rings such that for all $i \leq j$ the transition map $R_{i} \rightarrow R_{j}$ is flat. Then the direct limit $R$ is Gröbnercoherent.

Proof. It is a standard fact that $R$ is graded-coherent ([So, Proposition 20]). Now let $\mathfrak{a}$ be a finitely generated ideal of $R$. Since $\mathfrak{a}$ is finitely generated, there is some $i \in I$ such that $\mathfrak{a}$ is the extension of an ideal $\mathfrak{a}^{\prime} \subset R_{i}$ along the map $R_{i} \rightarrow R$. Since this map is flat, we have $\mathfrak{a}=\mathfrak{a}^{\prime} \otimes_{R_{i}} R$, and so a Gröbner basis of $\mathfrak{a}^{\prime}$ gives one of $\mathfrak{a}$ by Proposition 5.2. Thus $\mathfrak{a}$ has a finite Gröbner basis, and so $R$ is Gröbner-coherent.

Example 5.4. A direct limit of noetherian graded rings with flat transition maps is Gröbnercoherent. In particular, a polynomial ring (with any cardinality of variables) over a noetherian coefficient ring is Gröbner-coherent.

\section{Relation BetweEn DiffEREnt notions of COHEREnCE}

As we have seen, the following implications hold:

$$
\text { Gröbner-coherent } \Longrightarrow \text { coherent } \Longrightarrow \text { graded-coherent }
$$

We now show that both implications are strict.

First, let $\mathbf{k}$ be a coherent ring such that $\mathbf{k}[x]$ is not coherent. By [So, Proposition 18], such a ring exists: in fact, $\mathbf{k}$ can be taken to be a countable direct product of $\mathbf{Q} \llbracket t, u \rrbracket$ 's. It is easy to show that $\mathbf{k}[x]$ is graded-coherent (this also follows from [NS, §4.5]). Thus $\mathbf{k}[x]$ is an example of a ring that is graded-coherent but not coherent.

Next, let $\mathbf{k}$ be a valuation ring with non-archimedean valuation group. Thus, letting $v$ be the valuation on $\mathbf{k}$, there exist non-zero $a, b \in \mathbf{k}$ with $v(a)>0$ and $v(b)>n v(a)$ for all $n \in \mathbf{N}$. We claim that $\mathbf{k}[x]$ is coherent but not Gröbner-coherent. The coherence of $\mathbf{k}[x]$ follows from [RG, pg. 25] (or see [Gl, Theorem 7.3.3]). Now consider the ideal $I=(a x+1, b)$ in $\mathbf{k}[x]$. It is easy to see that the degree zero piece of $\operatorname{in}(I)$ is the $\mathbf{k}$-ideal generated by elements of the form $b / a^{n}$ for $n \geq 0$, and is clearly not finitely generated. Hence $\operatorname{in}(I)$ is not finitely generated, completing the proof of the claim. (This example comes from [Ye, pg. 10].)

\section{REFERENCES}

[Gl] S. Glaz, Commutative coherent rings, Lecture Notes in Mathematics 137, Springer-Verlag, 1989.

[NS] R. Nagpal, A. Snowden, The module theory of divided power algebras, preprint.

[RG] M. Raynaud and L. Gruson, Critéres de platitude et de projectivité, Invent. Math. 13 (1971), 1-89.

[So] J. P. Soublin, Anneaux et modules cohérents, J. Algebra 15 (1970), 455-472

[Ye] I. Yengui, Algorithms for computing syzygies over $V\left[X_{1}, \ldots, X_{n}\right], V$ a valuation ring, http://epiphymaths.univ-fcomte.fr/fete-a-henri/Yengui-Besancon.pdf

Department of Mathematics, University of Chicago, Chicago, IL

E-mail address: nagpal@math.uchicago.edu

$U R L:$ http://math.uchicago.edu/ nagpal/

Department of Mathematics, University of Michigan, Ann Arbor, Mi

E-mail address: asnowden@umich.edu

URL: http://www-personal.umich.edu/ asnowden/ 